\title{
Unsteady Thin-Airfoil Theory Revisited: Application of a Simple Lift Formula
}

\author{
Tianshu Liu* \\ Western Michigan University, Kalamazoo, Michigan 49008 \\ and \\ Shizhao Wang,, \pm Xing Zhang, \\ State Key Laboratory of Nonlinear Mechanics, Chinese Academy of Sciences, 100190 Beijing, \\ People's Republic of China
}

DOI: $\underline{10.2514 / 1 . J 053439}$

The physical foundations of unsteady thin-airfoil theory are explored in the general framework of viscous flows. The thin-airfoil lift formula is derived by using the simple lift formula that contains the vortex lift and the lift associated with the fluid acceleration. From a broader perspective, the thin-airfoil lift formula could be applicable even when the flow around an airfoil is moderately separated, from which the classical von Kármán-Sears lift formula can be recovered as a reduced case. The quantitative relationship between boundary layer and lift generation is discussed. Direct numerical simulations of low-Reynolds-number flows over a flapping flat-plate airfoil are conducted to examine the accuracy and limitations of the thin-airfoil lift formula.

$A$
$C l$
$c$
$D_{\mathrm{bl}}$
$D_{\mathrm{out}}$
$\boldsymbol{F}$
$f$
$k$
$\boldsymbol{k}$
$L, L^{\prime}$
$L_{a}, L_{a}^{\prime}$
$L_{\mathrm{vor}}, L_{\mathrm{vor}}^{\prime}$
$\boldsymbol{l}$
$\boldsymbol{n}$
$\boldsymbol{n}^{\prime}$
$p$
$q_{\infty}$
$R e$
$S$
$T$
$t$
$U$
$\boldsymbol{u}$
$V_{f}$
$x$
$(X, Y, Z)$
$x_{\mathrm{ref}}$
$x$
$z_{c}$
$\alpha$

\section{Nomenclature}

$=$ heaving amplitude, $\mathrm{m}$

$=$ lift coefficient

$=$ wing chord, $\mathrm{m}$

$=$ boundary-layer domain

$=$ outer flow domain

$=$ aerodynamic force, $\mathrm{N}$

$=$ flapping frequency, $\mathrm{s}^{-1}$

$=\pi f c / U$, reduced frequency

$=$ unit vector normal to the freestream velocity

$=\quad$ lift or sectional lift, $\mathrm{N}$ or $\mathrm{N} \cdot \mathrm{m}^{-1}$

$=$ lift or sectional lift associated with the fluid acceleration, $\mathrm{N}$ or $\mathrm{N} \cdot \mathrm{m}^{-1}$

$=$ vortex lift or sectional vortex lift, $\mathrm{N}$ or $\mathrm{N} \cdot \mathrm{m}^{-1}$
Lamb vector, $\mathrm{m} \cdot \mathrm{s}^{-2}$

unit normal vector pointing outward from control boundary

$-n$

pressure, $\mathrm{Pa}$

dynamic pressure, $\mathrm{Pa}$

Reynolds number

wing area, $\mathrm{m}^{2}$

flapping period, $\mathrm{s}$

time, $\mathrm{s}$

incoming flow velocity, $\mathrm{m} \cdot \mathrm{s}^{-1}$

fluid velocity, $\mathrm{m} \cdot \mathrm{s}^{-1}$

control volume

coordinate along the airfoil cord, $\mathrm{m}$

coordinates in direct numerical simulation, $\mathrm{m}$

reference location, $\mathrm{m}$

$\left(x-x_{\mathrm{LE}}\right) / c$, normalized coordinate

vertical position of airfoil center, $\mathrm{m}$

angle of attack, deg
Received 2 March 2014; revision received 25 June 2014; accepted for publication 1 August 2014; published online 31 October 2014. Copyright (C) 2014 by the American Institute of Aeronautics and Astronautics, Inc. All rights reserved. Copies of this paper may be made for personal or internal use, on condition that the copier pay the $\$ 10.00$ per-copy fee to the Copyright Clearance Center, Inc., 222 Rosewood Drive, Danvers, MA 01923; include the code 1533-385X/14 and $\$ 10.00$ in correspondence with the CCC.

*Professor, Department of Mechanical and Aerospace Engineering; tianshu.liu@wmich.edu. Senior Member AIAA (Corresponding Author).

${ }^{\dagger}$ Assistant Professor, Institute of Mechanics.

${ }^{\ddagger}$ Associate Professor, Institute of Mechanics.

\$Professor, Institute of Mechanics.

$\begin{array}{lll}\Gamma & =\text { circulation, } \mathrm{m}^{2} \cdot \mathrm{s}^{-1} \\ \gamma & = & \text { vortex sheet strength, } \mathrm{m} \cdot \mathrm{s}^{-1} \\ \gamma_{1} & = & \text { unsteady vortex sheet strength, } \mathrm{m} \cdot \mathrm{s}^{-1} \\ \gamma_{w} & = & \text { wake vortex sheet strength, } \mathrm{m} \cdot \mathrm{s}^{-1} \\ \gamma_{0} & = & \text { quasi-steady vortex sheet strength, } \mathrm{m} \cdot \mathrm{s}^{-1} \\ \partial B & = & \text { solid boundary of the body }(\mathrm{wing} \text { ) domain } \\ \partial B_{\mathrm{bl}} & = & \text { boundary-layer edge } \\ \rho & = & \text { fluid density, } \mathrm{kg} \cdot \mathrm{m}^{-3} \\ \Sigma & = & \text { outer surface of a control volume } \\ \tau & = & \text { skin friction, } \mathrm{N} \cdot \mathrm{m}^{-2} \\ \phi & = & \text { velocity potential, } \mathrm{m}^{2} \cdot \mathrm{s}^{-1} \\ \omega & = & \text { vorticity, } \mathrm{s}^{-1}\end{array}$

\section{Introduction}

$\mathbf{T}$ HIN-AIRFOIL theory originally developed by Munk [1] uses a vortex sheet in a potential flow to model an actual flow over a thin airfoil, in which the Kutta condition is imposed at the trailing edge to calculate the lift and moment. The higher-order approximation of thin-airfoil theory was studied by Lighthill [2]. The pioneering studies on unsteady thin-airfoil theory were conducted by Wagner [3], Küssner [4], Theodorsen [5], and von Kármán and Sears [6]. Recently, because of the renewed interests in active flutter control and flapping flight, considerable efforts have been made to apply thin-airfoil theory to unsteady flows associated with aeroelastic, flapping, and flexible wings [근 13]. The formulation given by von Kármán and Sears [6] based on the application of the vortex impulse is particularly insightful because the unsteady lift is explicitly expressed as a sum of the quasi-steady Kutta-Joukowski lift, the added-mass lift, and the wake-induced contribution. The wake-induced term leads to the so-called deficiency preventing the instantaneous lift from attaining the quasi-steady state immediately. Interestingly, the von KármánSears formulation remains the same when the nonlinear effects induced by the wake are incorporated by McCune and Tavares [14]. This implies that the von Kármán-Sears lift formula could be more generic in terms of its physical foundations.

The physical foundations of thin-airfoil theory were discussed by Glauert [15]. His argument is that a vortex sheet is a limiting model of a boundary layer on the airfoil surface as the viscosity approaches to zero and the integration of the vortex sheet strength is equal to the magnitude of the circulation generating the lift. The relationship between unsteady boundary-layer vorticity and bound-vortex sheet strength was further discussed by Sears [16] along with the generalized Kutta-Joukowski condition for a blunt trailing edge. It is clear that a bound-vortex sheet in thin-airfoil theory represents the airfoil plus its boundary layer, and the circulation is intimately 


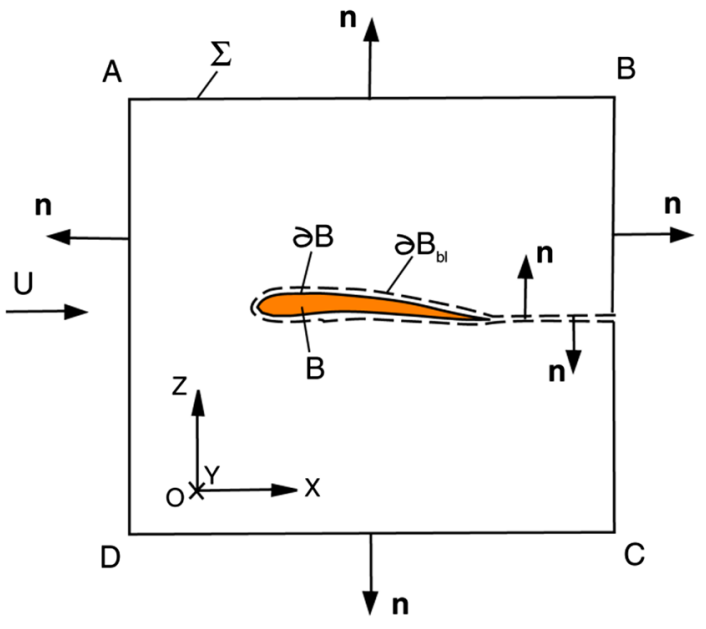

Fig. 1 Rectangular control volume.

connected with the boundary-layer. It is proposed by Sears [16] that the lift and moment are calculated by using thin-airfoil theory, and the circulation is determined by boundary-layer calculation such that thin-airfoil theory could be applied to a body with boundary-layer separation near the blunt trailing edge. Although these conceptual arguments are physically compelling, a derivation of unsteady thinairfoil theory from the Navier-Stokes (NS) equations has not been systematically given in the general framework of viscous flows. The feasible derivation should be based on a general force expression for viscous flows.

In an incompressible viscous flow, as shown in Fig. 1, the force acting on a solid body is given by

$$
\boldsymbol{F}=-\oint_{\partial B}(-p \boldsymbol{n}+\boldsymbol{\tau}) \mathrm{d} S=-\rho \int_{V_{f}} \boldsymbol{a} \mathrm{d} V+\oint_{\Sigma}(-p \boldsymbol{n}+\boldsymbol{\tau}) \mathrm{d} S
$$

where $p$ is the pressure, $\boldsymbol{\tau}$ is the surface shear-stress vector, $\boldsymbol{a}=$ $\mathrm{D} u / \mathrm{D} t$ is the acceleration, $\rho$ is the fluid density, $\partial B$ denotes a solid boundary of the body (wing) domain $B, V_{f}$ denotes the control volume of fluid, $\Sigma$ denotes an outer control surface in which the body is enclosed, and $\boldsymbol{n}$ is the unit normal vector pointing to the outside of a control surface. By using the equation $\boldsymbol{a}=\partial \boldsymbol{u} / \partial t+$ $\boldsymbol{\omega} \times \boldsymbol{u}+\nabla\left(q^{2} / 2\right)$, Eq. (1) becomes

$$
\begin{aligned}
\boldsymbol{F}= & \rho \int_{V_{f}} \boldsymbol{u} \times \boldsymbol{\omega} \mathrm{d} V-\rho \int_{V_{f}} \frac{\partial \boldsymbol{u}}{\partial t} \mathrm{~d} V-\oint_{\Sigma}\left(p+\rho q^{2} / 2\right) \boldsymbol{n} \mathrm{d} S \\
& +\oint_{\Sigma} \boldsymbol{\tau} \mathrm{d} S-\rho \oint_{\partial B}\left(q^{2} / 2\right) \boldsymbol{n} \mathrm{d} S
\end{aligned}
$$

where $\boldsymbol{u}$ is the velocity, $\boldsymbol{\omega}$ is the vorticity, and $q=|\boldsymbol{u}|$. The first term in the right-hand side (RHS) of Eq. (2) is a volume integral of the Lamb vector $\boldsymbol{l}=\boldsymbol{u} \times \boldsymbol{\omega}$ that represents the vortex force. The second term is a volume integral of the local acceleration of fluid, representing the unsteady inertial effect induced by a moving solid body and self-excited unsteady flow itself. The third and fourth terms are the surface integrals of the total pressure $P=p+\rho q^{2} / 2$ and the surface shear stress on the control surface $\Sigma$. The fifth term is the boundary term of the kinetic energy.

Because the static pressure $p$ is difficult to calculate and measure, the third term related to $p$ in Eq. (2) should be transformed to the terms related to the velocity that is more measurable. Different approaches have been used to deal with the troublesome pressure term, which leads to various force expressions [17-25]. However, for a general control surface, eliminating the pressure term usually results in more complicated expressions in which the physical meanings and relative contributions of some terms cannot be easily elucidated. The complicated forms of these expressions are not readily used to derive classical unsteady thin-airfoil theory, particularly the von Kármán-Sears unsteady airfoil theory. Recently,
Wang et al. [26] circumvented this pressure problem by selecting a rectangular control volume to obtain a very simple but sufficiently accurate lift formula. It is found that, for a rectangular control surface whose upper and lower faces are sufficiently far away from a wing, the contributions of the third and fourth terms in the RHS of Eq. (2) to the lift generation can be neglected. Thus, the lift can be approximately decomposed into the two dominant terms: the vortex force and the fluid acceleration. This leads to the simple lift formula that is useful in an analysis of the connection between vortical structures and lift generation in complex unsteady flows associated with flapping wings. The accuracy of the simple lift formula has been evaluated via direct numerical simulation (DNS) for unsteady low-Reynoldsnumber flows [26].

The objective of this work is to explore the physical foundations of unsteady thin-airfoil theory from a perspective of viscous flow theory and derive the thin-airfoil lift formula and the classical von KármánSears lift formula as a reduced case by using the simple lift formula. The development of this work is briefly outlined as follows. First, for a two-dimensional (2-D) flow over a thin airfoil, the flowfield is decomposed into a boundary layer (viscous flow region) and an outer potential flow. By applying the simple lift formula to this case, the thin-airfoil lift formula is given, which contains the vortex lift and the added-mass lift. In this reduction, the boundary layer plus the airfoil is naturally reduced to a vortex sheet as a key element in unsteady thin-airfoil theory. Then, to incorporate the wake effect into the theory, a decomposition of the vortex sheet strength into the quasisteady part without considering the wake effect and the unsteady part induced by the wake. Therefore, a triple decomposition of the lift is obtained, where the first term is the quasi-steady vortex lift (the Kutta-Joukowski lift), the second term is the added-mass lift, and the third term is the wake-induced term. This is a generalized version of the von Kármán-Sears lift formula with a general wake model. For the specific Green's function given by von Kármán and Sears [6] in the wake integral, the classical von Kármán-Sears lift formula is recovered. The relationship between a boundary layer and lift generation is discussed. Further, DNSs of low-Reynolds-number flows over a flapping flat-plate airfoil are conducted to examine the accuracy and limitations of the thin-airfoil lift formula in comparison the simple lift formula.

\section{Simple Lift Formula}

For a rectangular outer control surface $\Sigma$ whose upper and lower faces are sufficiently far away from the wing, the contributions of the third and fourth terms in the RHS of Eq. (2) to the lift generation can be neglected. Therefore, the simple lift formula (SLF) given by Wang et al. [26] is expressed in the two dominant terms, i.e.,

$$
L \approx L_{\mathrm{vor}}+L_{a}
$$

In Eq. (3), the vortex lift is given by

$$
L_{\mathrm{vor}}=\rho \boldsymbol{k} \cdot \int_{V_{f}} \boldsymbol{u} \times \boldsymbol{\omega} \mathrm{d} V
$$

where $\boldsymbol{k}$ is the unit vector normal to the freestream velocity. The lift associated with the fluid acceleration becomes

$$
\begin{aligned}
L_{a} & =-\rho \boldsymbol{k} \cdot \int_{V_{f}} \frac{\partial \boldsymbol{u}}{\partial t} \mathrm{~d} V-\rho \boldsymbol{k} \cdot \oint_{\partial B}\left(|\boldsymbol{u}|^{2} / 2\right) \boldsymbol{n} \mathrm{d} S \\
& =-\rho \boldsymbol{k} \cdot \frac{\mathrm{d}}{\mathrm{d} t} \int_{V_{f}} \boldsymbol{u} \mathrm{d} V+\rho \boldsymbol{k} \cdot \oint_{\Sigma} \boldsymbol{n} \cdot\left[\frac{1}{2}|\boldsymbol{u}|^{2} \boldsymbol{I}-\boldsymbol{u} \boldsymbol{u}\right] \mathrm{d} S \\
& =-\rho \boldsymbol{k} \cdot \frac{\mathrm{d}}{\mathrm{d} t} \int_{V_{f}} \boldsymbol{u} \mathrm{d} V
\end{aligned}
$$

where $\boldsymbol{I}$ is the identity tensor, and $\boldsymbol{n}$ is the unit normal vector pointing outward from the control boundary. Because $\boldsymbol{k} \cdot \boldsymbol{n}=0$ on the vertical faces and $\boldsymbol{k} \cdot \boldsymbol{u} \rightarrow \boldsymbol{k} \cdot \boldsymbol{i} U=0$ on the top and bottom faces, the term $\boldsymbol{k} \cdot \boldsymbol{n} \cdot\left(0.5|\boldsymbol{u}|^{2} \boldsymbol{I}-\boldsymbol{u u}\right)$ approaches to zero. It has been 
demonstrated that the SLF is sufficiently accurate for complex unsteady viscous flows generated by flapping wings [26]. Because of the formal simplicity and physical clarity of the SLF, it is particularly useful to evaluate the contributions of distinct vortical structures to the lift in unsteady flows. In a limiting case where a moving body is in a completely inviscid irrotational flow with $\boldsymbol{u}=\nabla \phi, L_{a}$ is interpreted as the added-mass force projected on the direction of $\boldsymbol{k}$. In this case, $L_{a}$ is expressed as $L_{a}=m_{a} \mathrm{~d} U_{b} / \mathrm{d} t$, where the added mass is defined as $m_{a} \approx-\rho l_{\text {ref }} \int_{\partial B} \bar{\phi}(\boldsymbol{k} \cdot \boldsymbol{n}) \mathrm{d} S, \phi=\phi\left[U_{b}(t) l_{\text {ref }}\right]^{-1}$ is the normalized velocity potential, and $U_{b}(t)$ and $l_{\text {ref }}$ the velocity and the reference length of the body, respectively.

\section{Thin-Airfoil Lift Formula}

A 2-D attached flowfield over a thin airfoil can be decomposed into the outer potential flow and the boundary layer (or the viscous flow region). Therefore, the velocity is expressed as $\boldsymbol{u}=$ $\nabla \phi H\left(x \in D_{\text {out }}\right)+\boldsymbol{u} H\left(\boldsymbol{x} \in D_{\mathrm{bl}}\right)$, where $\phi$ is the velocity potential, $D_{\text {out }}$ and $D_{\mathrm{bl}}$ denote the outer flow domain and the boundary-layer domain $\left(V_{f}=D_{\text {out }}+D_{\mathrm{bl}}\right)$, respectively, and the Heaviside function is defined as $H(x \in D)=1$ and $H(x \notin D)=0$. The boundary-layer edge is denoted by $\partial B_{\mathrm{bl}}$ that separates the two domains. In this case, the vortex lift is solely contributed by the boundary layer. For a 2-D attached flow, according to the SLF, the lift per unit span is given by

$$
L^{\prime}=L_{\mathrm{vor}}^{\prime}+L_{a}^{\prime} \approx \rho U_{\mathrm{eff}} \Gamma+\rho \frac{\mathrm{d}}{\mathrm{d} t} \int_{\partial B_{\mathrm{b} 1}} \phi\left(\boldsymbol{k} \cdot \boldsymbol{n}^{\prime}\right) \mathrm{d} S
$$

where $\Gamma=\left\langle\omega_{y}\right\rangle_{D} D$ is the circulation, $U_{\text {eff }}=\left\langle u_{\text {eff }}\right\rangle_{D}$ is the areaaveraged effective velocity, $u_{\text {eff }}=u \omega_{y} /\left\langle\omega_{y}\right\rangle_{D},\left\langle\omega_{y}\right\rangle_{D}$ is the areaaveraged spanwise vorticity, $u$ is the velocity component in the $x$ direction, and $\boldsymbol{n}^{\prime}$ is the unit normal vector pointing outward from the wing surface $\left(\boldsymbol{n}^{\prime}=-\boldsymbol{n}\right)$. The domain-averaged operator $\langle\bullet\rangle_{D}=D^{-1} \int_{D} \bullet \mathrm{d} V$, and $D$ is a rectangular control domain in 2-D. For a bounded vorticity region (e.g., a boundary layer) that is much smaller than the control domain $D$, it is proven by Wang et al. [26] that the effective velocity is equal to the incoming flow velocity (i.e., $U_{\text {eff }}=U$ ), and thus the Kutta-Joukowski theorem $L_{\mathrm{vor}}^{\prime}=\rho U \Gamma$ is recovered. For a rectangular outer control surface $\Sigma$, as shown in Sec. VI, $L_{a}^{\prime}$ in Eq. (6) is approximately expressed as the time rate of the surface integral of the velocity potential on $\partial B_{\mathrm{bl}}$ by using the Gauss theorem. The underlying assumption for such an approximation is that the integral momentum of fluid in the boundary layer is much smaller than that in the inviscid outer flow in unsteady flows. In other words, $L_{a}^{\prime}$ in Eq. (6) mainly represents the added-mass lift associated with the unsteady outer flow induced by a moving wing, neglecting the unsteady effects of the viscous flow domain (e.g., the boundary layer) near the wing.

Further, for a thin airfoil, the circulation is given by the integral of the physical quantity $\gamma(x, t)$ along the coordinate $x$ on the chord line of the airfoil, i.e.,

$$
\Gamma=\int_{D_{\mathrm{bl}}} \omega_{y} \mathrm{~d} S=\int_{x_{\mathrm{LE}}}^{x_{\mathrm{TE}}} \gamma(x, t) \mathrm{d} x
$$

In Eq. (7), $\gamma(x, t)$ is defined as

$$
\gamma(x, t)=\int_{0}^{\delta^{+}} \omega_{y}^{+} \mathrm{d} n^{\prime+}+\int_{0}^{\delta^{-}} \omega_{y}^{-} \mathrm{d} n^{\prime-} \approx u_{x e}^{+}-u_{x e}^{-}
$$

where the superscripts "+" and "-" denote the quantities on the upper and lower surfaces of the thin airfoil, respectively; $\delta$ denotes the boundary-layer thickness; $n^{\prime}$ is the normal coordinate directing outward from the thin airfoil surface; and $u_{x e}^{+}-u_{x e}^{-}$is the velocity difference between the boundary-layer edges on the upper and lower surfaces. The function $\gamma(x, t)$ is a lumped model of the vorticity distribution on the airfoil surface. In the limiting case where the boundary layer becomes very thin as Reynolds number is increased, $\gamma(x, t)$ is interpreted as the strength of a vortex sheet in the classical thin-airfoil theory.
The added-mass lift per unit span is

$$
\begin{aligned}
L_{a}^{\prime} & \approx \rho \frac{\mathrm{d}}{\mathrm{d} t} \int_{\partial B_{\mathrm{bl}}} \phi\left(\boldsymbol{k} \cdot \boldsymbol{n}^{\prime}\right) \mathrm{d} S \approx \rho \frac{\mathrm{d}}{\mathrm{d} t} \int_{x_{\mathrm{LE}}}^{x_{\mathrm{TE}}}\left(\phi_{e}^{+}-\phi_{e}^{-}\right) \mathrm{d} x \\
& =\rho \frac{\mathrm{d}}{\mathrm{d} t} \int_{x_{\mathrm{LE}}}^{x_{\mathrm{TE}}}\left(x_{\mathrm{ref}}-x\right) \gamma(x, t) \mathrm{d} x
\end{aligned}
$$

where $x_{\mathrm{LE}}$ and $x_{\mathrm{TE}}$ are the leading-edge and trailing-edge locations, respectively, and the subscript " $e$ " denotes the boundary-layer edge. In the derivation of the second approximate equality in Eq. (9), it is assumed that $\left(\boldsymbol{k} \cdot \boldsymbol{n}^{\prime}\right) d S \approx d x$ in 2-D. Another approximation is that the effect of the unsteady boundary-layer edge $\partial B_{\mathrm{bl}}$ is neglected because $x_{\mathrm{LE}}$ and $x_{\mathrm{TE}}$ are treated as the time-independent variables. The effect of the unsteady boundary-layer edge $\partial B_{\mathrm{bl}}$ as a part of the added-mass force will be evaluated in Sec. VI. In the derivation of the last equality in Eq. (9), integration by parts is carried out, and the reference location $x_{\text {ref }}$ is introduced as a parameter when the mean value theorem is applied. The reference location $x_{\text {ref }}$ will be determined later in a classical flow across an accelerating flat plate in which $\bar{x}_{\text {ref }}=0.5$ is found. It is interesting that $L_{a}^{\prime}$ in Eq. (9) is reduced to the time rate of the vortex impulse (or vortex moment) in unsteady thin-airfoil theory.

Substitution of Eqs. (7) and (9) into Eq. (6) yields the thin-airfoil lift formula (TALF):

$$
\begin{aligned}
L^{\prime}(t) & =L_{\mathrm{vor}}^{\prime}(t)+L_{a}^{\prime}(t) \\
& \approx \rho U(t) c \int_{0}^{1} \gamma(\bar{x}, t) \mathrm{d} \bar{x}+\rho c^{2} \frac{\mathrm{d}}{\mathrm{d} t} \int_{0}^{1}\left(\bar{x}_{\mathrm{ref}}-\bar{x}\right) \gamma(\bar{x}, t) \mathrm{d} \bar{x}
\end{aligned}
$$

where $\bar{x}=\left(x-x_{\mathrm{LE}}\right) / c$ is the normalized coordinate, $\bar{x}_{\text {ref }}=$ $\left(x_{\text {ref }}-x_{\mathrm{LE}}\right) / c$ is the normalized reference location, and $c$ is the chord. The first and second terms in the RHS of in Eq. (10) are interpreted as the Kutta-Joukowski lift and the added-mass lift, respectively. The previous analysis shows how Eq. (3) is mathematically reduced to Eq. (10), in which a vortex sheet is considered as an idealized model of a boundary layer (or near-wall shear layer) for a thin airfoil. This supports the arguments made by Glauert [15] and Sears [16] on the physical foundations of thin-airfoil theory, in which a vortex sheet is a limiting model of a boundary layer on the airfoil surface as the viscosity approaches to zero. Sears [16] obtained Eq. (10) using the unsteady Bernoulli equation in the framework of inviscid flows but argued physically that it could be correct even when the boundary layer is separated. In fact, the aforementioned derivation of Eq. (10) can be extended to moderately separated flows by defining the limits $\delta^{+}$and $\delta^{-}$in the integral of the vorticity field [Eq. (8)] as the sufficiently large distances from the airfoil surface beyond the viscous flow region. In this sense, the whole separated flow plus an airfoil is vertically compressed into a vortex sheet with the strength $\gamma(x, t)$. When $\gamma(x, t)$ is calculated from the vorticity fields obtained from global velocity measurements and CFD, the nonlinear effects associated with viscous separated flows could be naturally incorporated in the TALF particularly in the vortex lift. This will be critically examined through DNS of low-Reynolds-number flows over a flapping flat-plate airfoil in Sec. VII.

\section{Wake Effect}

In the linear theory of aerodynamics, the formal solution of the thin-airfoil equation is first sought for $\gamma(x, t)$, and then Green's function in the wake-induced term is determined. The thin-airfoil equation is

$$
\frac{1}{2 \pi} \int_{0}^{1} \frac{\gamma(\bar{\xi}, t)}{\bar{\xi}-\bar{x}} \mathrm{~d} \bar{\xi}=F_{0}(\bar{x}, t)+F_{1}(\bar{x}, t)
$$

where $\bar{\xi}=\left(\xi-x_{\mathrm{LE}}\right) / c$ and $\bar{x}=\left(x-x_{\mathrm{LE}}\right) / c$ are the normalized coordinates; $c$ is the chord; and $F_{0}(\bar{x}, t)$ and $F_{1}(\bar{x}, t)$ are the quasisteady velocity normal to the airfoil surface associated with the wing kinematics and the normal velocity induced by the wake, 
respectively. The singular integrals in Eq. (11) and several other equations in this paper are in the sense of the Cauchy principal value. The specific forms of $F_{0}(\bar{x}, t)$ and $F_{1}(\bar{x}, t)$ are given by Katz and Plotkin [27]. The wake-induced normal velocity on the airfoil can be expressed as

$$
F_{1}(\bar{x}, t)=\int_{1}^{\infty} S(\bar{\xi} ; \bar{x}, t) \gamma_{w}(\bar{\xi}, t) \mathrm{d} \bar{\xi}
$$

where $S(\bar{\xi} ; \bar{x}, t)$ is related to the distribution of the wake vortices relative to the airfoil, and $\gamma_{w}$ is the strength of the wake vortex sheet.

From a standpoint of theoretical aerodynamics, to formally isolate the effect of the wake, a decomposition $\gamma=\gamma_{0}+\gamma_{1}$ is used, where $\gamma_{0}$ is the quasi-steady part without considering the effect of the wake vortex sheet, and $\gamma_{1}$ is the unsteady part induced by the wake. Therefore, using the solution of the Cauchy integral equation of the first kind [28] and imposing the Kutta condition $\gamma(\bar{x}=1, t)=0$, we have

$$
\begin{aligned}
& \gamma_{0}(\bar{x}, t)=\frac{2}{\pi \sqrt{\bar{x}(1-\bar{x})}} \int_{0}^{1} E(\bar{\xi} ; \bar{x}) F_{0}(\bar{\xi}, t) \mathrm{d} \bar{\xi} \\
& \gamma_{1}(\bar{x}, t)=\frac{2}{\pi \sqrt{\bar{x}(1-\bar{x})}} \int_{0}^{1} E(\bar{\xi} ; \bar{x}) F_{1}(\bar{\xi}, t) \mathrm{d} \bar{\xi}
\end{aligned}
$$

where

$$
E(\bar{\xi}, \bar{x})=\frac{\sqrt{\bar{\xi}(1-\bar{\xi})}}{\bar{x}-\bar{\xi}}-\frac{\sqrt{\bar{\xi}(1-\bar{\xi})}}{1-\bar{\xi}}
$$

It is noted that Eq. (11) is usually solved by using Glauert's Fourier series method $[15,2 \overline{7}]$. However, Eqs. (13) and (14) have a more compact form that is convenient for mathematical manipulation. Further, by using Eq. (12), the relation between $\gamma_{1}$ and $\gamma_{w}$ is given by

$$
\gamma_{1}(\bar{x}, t)=\int_{1}^{\infty} G\left(\bar{\xi}_{w} ; \bar{x}, t\right) \gamma_{w}\left(\bar{\xi}_{w}, t\right) \mathrm{d} \bar{\xi}_{w}
$$

where the Green's function is

$$
G\left(\bar{\xi}_{w} ; \bar{x}, t\right)=\frac{2}{\pi \sqrt{\bar{x}(1-\bar{x})}} \int_{0}^{1} E(\bar{\xi}, \bar{x}) S\left(\bar{\xi}_{w} ; \bar{\xi}, t\right) \mathrm{d} \bar{\xi}
$$

Substituting $\gamma=\gamma_{0}+\gamma_{1}$ into Eq. (10) (the TALF) and using Eq. (16) and an integral formula given by von Kármán and Sears [] [Eq. (트) in their paper] for the wake vortices traveling with the freestream velocity $U$, we have

$$
\begin{aligned}
L^{\prime}(t) \approx & \rho U(t) c \int_{0}^{1} \gamma_{0}(\bar{x}, t) \mathrm{d} \bar{x}+\rho c^{2} \frac{\mathrm{d}}{\mathrm{d} t} \int_{0}^{1}\left(\bar{x}_{\mathrm{ref}}-\bar{x}\right) \gamma_{0}(\bar{x}, t) \mathrm{d} \bar{x} \\
& +\rho U(t) c \int_{1}^{\infty} \gamma_{w}(\bar{\xi}, t) P(\bar{\xi}, t) \mathrm{d} \bar{\xi}
\end{aligned}
$$

where the relevant functions in the wake terms are defined as

$$
\begin{gathered}
P(\bar{\xi}, t)=\frac{\mathrm{d} H_{1}(\bar{\xi}, t)}{\mathrm{d} \bar{\xi}}+H_{2}(\bar{\xi}, t) \\
H_{1}(\bar{\xi}, t)=\int_{0}^{1}\left(\bar{x}_{\mathrm{ref}}-\bar{x}\right) G(\bar{\xi} ; \bar{x}, t) \mathrm{d} \bar{x} \\
H_{2}(\bar{\xi}, t)=\int_{0}^{1} G(\bar{\xi} ; \bar{x}, t) \mathrm{d} \bar{x}
\end{gathered}
$$

In Eq. (18), the first term in the RHS is the quasi-steady vortex lift, the second term is the added-mass lift, and the third term describes the effect of the wake on the lift, which explicitly depends on the strength distribution of the wake vortex sheet. Clearly, Eq. (18) is a reduced form of the TALF when the decomposition $\gamma=\gamma_{0}+\bar{\gamma}_{1}$ is introduced and the solution of the thin-airfoil equation is applied. On the other hand, Eq. (18) is considered as a generalized form of the von Kármán-Sears lift formula, which will be discussed in Sec. V.

The total circulation condition $\Gamma_{0}(t)+\Gamma_{w}(t)+\Gamma_{1}(t)=\overline{0}$ gives the following integral equation for $\gamma_{w}$ :

$$
\Gamma_{0}(t)=-\int_{1}^{\infty} \gamma_{w}(\bar{\xi}, t) Q(\bar{\xi}, t) \mathrm{d} \bar{\xi}
$$

where $Q(\bar{\xi}, t)=H_{2}(\bar{\xi}, t)+1$ is the kernel, and

$$
\begin{aligned}
\Gamma_{0}(t) & =\int_{0}^{1} \gamma_{0}(\bar{x}, t) \mathrm{d} \bar{x}, \quad \Gamma_{1}(t)=\int_{0}^{1} \gamma_{1}(\bar{x}, t) \mathrm{d} \bar{x}, \\
\Gamma_{w}(t) & =\int_{1}^{\infty} \gamma_{w}(\bar{\xi}, t) \mathrm{d} \bar{\xi}
\end{aligned}
$$

For a specific form $Q=\sqrt{\bar{\xi} /(\bar{\xi}-1)}$ considered by von Kármán and Sears [6], Eq. (22) recovers the Wagner integral equation. When the wake vortices are carried away with the freestream velocity, Eq. (22) has an approximate convolution form

$$
\Gamma_{0}(\sigma)=-\int_{0}^{\sigma(t)} \gamma_{w}(s) Q(\sigma-s) \mathrm{d} s
$$

where $s=\sigma(t)-\bar{\xi}+1, \sigma(t)=R(t) / c$, and $R(t)=\int{ }_{0}^{t} U(t) \mathrm{d} t$ is the downstream extremity of the wake. Therefore, a formal solution of Eq. (24) is

$$
\gamma_{w}(\sigma)=\int_{0}^{\infty} \Gamma_{0}\left(\sigma^{\prime}\right) W_{1}\left(\sigma-\sigma^{\prime}\right) \mathrm{d} \sigma^{\prime}
$$

where $W_{1}=-L^{-1}[1 / L(Q)]$ is a Green's function, and $L$ and $L^{-1}$ denote the Laplace transform and the inverse Laplace transform, respectively. The third term in the RHS of Eq. (18) that represents the wake effect can be formally written as

$$
\begin{aligned}
L_{w}^{\prime}(t) & =\rho U(t) c \int_{0}^{\sigma} \gamma_{w}(s) P(\sigma-s) \mathrm{d} s \\
& =\rho U(t) c \int_{0}^{\infty} \Gamma_{0}\left(\sigma^{\prime}\right) W_{2}\left(\sigma-\sigma^{\prime}\right) \mathrm{d} \sigma^{\prime}
\end{aligned}
$$

where $W_{2}=-L^{-1}[L(P) / L(Q)]$ is a Green's function that can be evaluated by using contour integral [29]. It is noted that the previous treatment of the wake effect based on the integral equation is just formally neat. From a computational viewpoint, however, discrete vortex methods are much easier and more effective to evaluate the wake effect [27].

\section{Von Kármán-Sears Lift Formula}

The vortex impulse was originally used by von Kármán and Sears [6] to calculate the unsteady lift. In fact, Eq. (18) can be directly used to calculate the unsteady lift. When a rigid wake vortex sheet is aligned and traveled with the freestream velocity, von Kármán and Sears [6] gave a specific time-independent Green's function:

$$
G(\bar{\xi} ; \bar{x})=\frac{1}{\pi} \overline{\bar{\xi}-\bar{x}} \sqrt{\frac{1-\bar{x}}{\bar{x}}} \sqrt{\frac{\bar{\xi}}{\bar{\xi}-1}}
$$

Substitution of Eq. (27) into Eqs. (20) and $(\underline{21)})(\bar{\xi}>1)$ yields

$$
H_{1}(\bar{\xi})=\left(\bar{x}_{\text {ref }}-0.5\right) \sqrt{\frac{\bar{\xi}}{\bar{\xi}-1}}+\left(\bar{\xi}-\bar{x}_{\text {ref }}\right)-\sqrt{\bar{\xi}(\bar{\xi}-1)}
$$




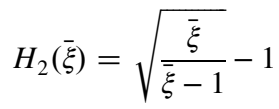

Therefore, the Green's function $P(\bar{\xi})$ in Eq. $(\underline{19})$ is

$$
P(\bar{\xi})=\frac{0.5-\bar{x}_{\text {ref }}}{4 \sqrt{\bar{\xi}(\bar{\xi}-1)^{3}}}+\frac{1}{2 \sqrt{\bar{\xi}(\bar{\xi}-1)}}
$$

The reference location $\bar{x}_{\text {ref }}$ can be determined based on a classical solution for the cross flow over an accelerating flat plate in fluid. In this case, the vortex sheet strength is $\gamma=-4 w(t) \eta / \sqrt{1-4 \eta^{2}}$, where $w(t)$ is the velocity of the flat plate and $\eta=\bar{x}-0.5$ [27]. According to Eq. (10), the added-mass force on the flat plate is

$$
L_{a}^{\prime}(t)=\rho c^{2} \frac{\mathrm{d}}{\mathrm{d} t} \int_{-1 / 2}^{1 / 2}\left(\eta_{\mathrm{ref}}-\eta\right) \gamma(\eta, t) \mathrm{d} \eta=\frac{\pi}{4} \rho c^{2} \dot{w}-4 \rho c^{2} \dot{w} \eta_{\mathrm{ref}} I_{0}
$$

where $\dot{w}=\mathrm{d} w / \mathrm{d} t$ is the acceleration, $\eta_{\text {ref }}=\bar{x}_{\text {ref }}-0.5$ is the reference location relative to the midpoint, and $I_{0}$ is a divergent integral given by

$$
I_{0}=\int_{-1 / 2}^{1 / 2} \frac{\eta}{\sqrt{1-4 \eta^{2}}} \mathrm{~d} \eta
$$

If Eq. (31) is consistent with the classical solution $L_{a}^{\prime}(t)=$ $(\pi / 4) \rho c^{2} \overline{\dot{w}}$, the necessary condition is $\eta_{\text {ref }}=0$ or $\bar{x}_{\text {ref }}=0.5$ such that the term with $I_{0}$ is nullified. Therefore, when the reference location is at the midpoint of a thin airfoil $\left(\bar{x}_{\text {ref }}=0.5\right)$, the first term of the RHS of Eq. (30) vanishes. As a result, Eq. (18) is reduced to the von Kármán-Sears lift formula:

$$
\begin{aligned}
L^{\prime}(t)= & \rho U(t) c \Gamma_{0}+\rho c^{2} \frac{\mathrm{d}}{\mathrm{d} t} \int_{0}^{1}(0.5-\bar{x}) \gamma_{0}(\bar{x}, t) \mathrm{d} \bar{x} \\
& +\rho U(t) c \int_{1}^{\infty} \gamma_{w}(\bar{\xi}, t) \frac{0.5 d \bar{\xi}}{\sqrt{\bar{\xi}(\bar{\xi}-1)}}
\end{aligned}
$$

Thus, the von Kármán-Sears vortex impulse formulation in the inviscid-fluid case is consistent with Eqs. (3) and (10), and the von Kármán-Sears lift formula is a reduced form of the TALF when the specific wake model is used for a thin airfoil.

\section{Boundary Layer and Lift Generation}

The relationship between a boundary layer and lift generation is further discussed by using the SLF. For a flow over a wing (or an airfoil in 2-D), as shown in Fig. 1, the flowfield is decomposed into the outer potential flow domain $\bar{D}_{\text {out }}$ and the boundary-layer domain $D_{\mathrm{bl}}$ that are separated by the boundary-layer edge $\partial B_{\mathrm{bl}}$. Therefore, the velocity is expressed as $\boldsymbol{u}=\nabla \phi H\left(\boldsymbol{x} \in D_{\text {out }}\right)+\boldsymbol{u} H\left(\boldsymbol{x} \in D_{\mathrm{bl}}\right)$, where $\phi$ is the velocity potential, and the Heaviside function is defined as $H(x \in D)=1$ and $H(x \notin D)=0$. In this case, the vortex lift is only contributed by the boundary layer. Under the boundarylayer approximation, the Lamb vector is $\boldsymbol{l}=\boldsymbol{u} \times \boldsymbol{\omega} \approx$ $0.5 \boldsymbol{n}^{\prime} \partial\left(u_{1}^{2}+u_{2}^{2}\right) / \partial x_{3}$, where $\left(u_{1}, u_{2}\right)$ is the velocity vector along the surface, $x_{3}$ is the coordinate normal to the surface, and $\boldsymbol{n}^{\prime}$ is the unit normal vector pointing outward from the wing surface $\left(\boldsymbol{n}^{\prime}=-\boldsymbol{n}\right)$. Therefore, the vortex lift is given by

$$
L_{\mathrm{vor}}=\rho \boldsymbol{k} \cdot \int_{D_{\mathrm{b} 1}} \boldsymbol{u} \times \boldsymbol{\omega} \mathrm{d} V \approx 0.5 \rho \boldsymbol{k} \cdot \int_{\partial B_{\mathrm{b} 1}} \boldsymbol{n}^{\prime}\left(u_{1 e}^{2}+u_{2 e}^{2}\right) \mathrm{d} S
$$

where $\left(u_{1 e}, u_{2 e}\right)$ is the velocity vector at the boundary-layer edge $\partial B_{\mathrm{bl}}$ along the surface. Substitution of Bernoulli's equation $0.5 \rho\left(u_{1}^{2}+\right.$ $\left.u_{2}^{2}\right)=p_{0}-p_{e}-\rho \partial \phi / \partial t$ into Eq. (34) leads to

$$
L_{\mathrm{vor}} \approx-\boldsymbol{k} \cdot \int_{\partial B_{\mathrm{b} 1}} \boldsymbol{n}^{\prime} p_{e} \mathrm{~d} S-\rho \boldsymbol{k} \cdot \int_{\partial B_{\mathrm{b} 1}} \boldsymbol{n}^{\prime} \frac{\partial \phi}{\partial t} \mathrm{~d} S
$$

Because the integral momentum of fluid in the boundary layer is much smaller than that in the outer flow, the lift associated with the fluid acceleration is

$$
L_{a} \approx-\rho \boldsymbol{k} \cdot \frac{\mathrm{d}}{\mathrm{d} t} \int_{D_{\text {out }}} \nabla \phi \mathrm{d} V=-\rho \boldsymbol{k} \cdot \frac{\mathrm{d}}{\mathrm{d} t} \int_{\Sigma+\partial B_{\mathrm{bl}}} \phi \boldsymbol{n} \mathrm{d} S
$$

where $\Sigma$ denotes the rectangular outer control surface, $\partial B_{\mathrm{bl}}$ denotes the edge boundary of the boundary-layer domain, and $\boldsymbol{n}$ is the unit normal vector of the boundary. As shown in Fig. 1, the surface integral on the rectangular surface $\Sigma$ in Eq. (36) vanishes because $\boldsymbol{k} \cdot \boldsymbol{n}=0$ on the vertical faces $\mathrm{AD}$ and $\mathrm{BC}$, and $\mathrm{d} \phi / \mathrm{d} t$ on the upper and lower faces $\mathrm{AB}$ and $\mathrm{DC}$ is not only decreased rapidly in the far field but also canceled out. The use of the Leibniz integral rule yields

$$
L_{a} \approx-\rho \boldsymbol{k} \cdot \frac{\mathrm{d}}{\mathrm{d} t} \int_{\partial B_{\mathrm{bl}}} \phi \boldsymbol{n} \mathrm{d} S=\rho \boldsymbol{k} \cdot \int_{\partial B_{\mathrm{bl}}} \boldsymbol{n}^{\prime} \frac{\partial \phi}{\partial t} \mathrm{~d} S+L_{a 0}
$$

where the term associated with the unsteady boundary-layer edge $\partial B_{\mathrm{bl}}$ is given by

$$
L_{a 0}=\rho \int_{\partial B_{\mathrm{bl}}}\left[(\phi \boldsymbol{k} \cdot \nabla) \boldsymbol{u}_{b}+\nabla \cdot(\phi \boldsymbol{k}) \boldsymbol{u}_{b}-\left(\nabla \cdot \boldsymbol{u}_{b}\right)(\phi \boldsymbol{k})\right] \cdot \boldsymbol{n}^{\prime} \mathrm{d} S
$$

$\boldsymbol{u}_{b}$ is the moving velocity of $\partial B_{\mathrm{bl}}$ as a geometric boundary, and $\boldsymbol{n}^{\prime}=-\boldsymbol{n}$ on $\partial B_{\mathrm{bl}}$ directs outward from the boundary layer. According to Eq. (37), $L_{a}$ is interpreted as the added-mass lift in this case. Based on Eqs. (35) and (37), the lift $L \approx L_{\mathrm{vor}}+L_{a}$ is expressed as

$$
L \approx-\boldsymbol{k} \cdot \int_{\partial B_{\mathrm{bl}}} \boldsymbol{n}^{\prime} p_{e} \mathrm{~d} S+L_{a 0}
$$

The first term in the RHS of Eq. (39) is the contribution calculated by integrating the pressure on the boundary-layer edge $\partial B_{\mathrm{bl}}$ around the wing. The second term represents the added-mass effect induced by the unsteady boundary-layer edge $\partial B_{\mathrm{bl}}$. The previous analysis gives a direct link between a boundary layer and lift generation. Because a boundary layer is also the source of the skin friction drag, the skin friction drag could be considered in a certain sense as a cost of generating the lift in addition to the induced drag.

\section{Flapping Flat-Plate Airfoil}

\section{A. Kinematics and Numerical Method}

To evaluate the accuracy and limitations of the thin-airfoil lift formula, the 2-D flows over a flapping flat-plate airfoil at a low Reynolds number are investigated through DNS. The plate has a chord length $c$ and zero thickness, heaving vertically and rotating around the center of the plate in a uniform flow. The Cartesian system $O-X Y Z$ fixed in the space is used in DNS. As shown in Fig. 2 , the $X$ axis directs downstream in parallel to the freestream, the $Z$ axis is

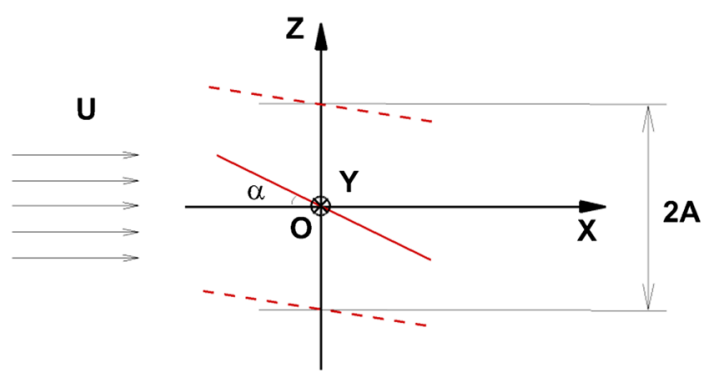

Fig. 2 Flapping flat-plate airfoil and coordinate system in DNS. 
Table 1 Kinematical parameters of a flapping flat-plate airfoil

\begin{tabular}{cccccc}
\hline \hline Case & $k=\pi f c / U$ & $A / c$ & $\alpha_{0}, \mathrm{deg}$ & $\alpha_{m}, \operatorname{deg}$ & $z_{c 0} / c$ \\
\hline $\mathrm{A}$ & $0.06 \pi$ & 0.025 & 1 & 3 & 0 \\
$\mathrm{~B}$ & $0.6 \pi$ & 0.025 & 1 & 3 & 0 \\
$\mathrm{C}$ & $0.06 \pi$ & 0.25 & 10 & 30 & 0 \\
$\mathrm{D}$ & $0.6 \pi$ & 0.25 & 10 & 30 & 0 \\
\hline \hline
\end{tabular}

perpendicular to the freestream pointing upward, and the $Y$ axis is normal to the $X Z$ plane along the wingspan. The kinematics of the flapping plate is described by the pitching angle (the geometrical angle of attack, or AOA) and the vertical position of the center of the plate, i.e.,

$$
\begin{gathered}
\alpha=\alpha_{0}+\alpha_{m} \cos (2 \pi f t) \\
z_{c}=z_{c 0}+A \sin (2 \pi f t)
\end{gathered}
$$

where $\alpha$ is the instantaneous AOA, $\alpha_{0}$ is the time-averaged AOA, and $\alpha_{m}$ is the pitching amplitude. $z_{c}$ is the vertical position of the center of the plate, $z_{c 0}$ is the time-averaged vertical position of the center, and $A$ is the heaving amplitude. $f$ is the pitching and/or heaving frequency. The reduced frequency of the flapping wing is defined as $k=$ $\pi f c / U$. The kinematic parameters in the four cases studied in DNS are given in Table 1.

The flow around the flapping airfoil is determined by the incompressible NS equations. The Reynolds number based on the chord length of the plate and uniform upstream flow is $R e=$ $U c / \nu=300$. The unsteady flows with the moving boundaries are simulated by using the immersed boundary method based on the discrete stream function formulation developed by Wang and Zhang [30]. In this method, the discrete stream function method (or exact projection method, null space method) proposed by Chang et al. [31] is used to solve the NS equations on a Cartesian Eulerian grid. The geometry and kinematics of the moving boundaries are described by a Lagrangian grid. The effects of the boundaries on the flow are represented by adding a force term in the NS equations. The force term is determined by solving a linear system regarding the interpolation between the Lagrangian and Eulerian grid points. The validations and the details of the numerical method can be found in our previous work for various flows $[26,30]$.

The computational domain used in the present work is $[-12,20] \times$ $[-16,16]$ in the streamwise $(X)$ direction and vertical $(Z)$ direction for 2-D flows. The unstructured Cartesian mesh with the hanging nodes is used in the simulations to refine the mesh around the plate. The minimum grid size is $d h=0.02$, and the maximum grid size is $d h=0.32$. The time step is selected to keep the maximum CourantFriedrichs-Lewy number at 0.5 . The independence of the lift coefficient on the grid resolution for the flow over the flapping plate has been examined. In all the simulations, the uniform upstream flow is set at the inlet, and the free convection flow is set at the outlet. The nonslip boundary condition is specified at the surface of the plate/ rectangular wing. The zero-shear slip-wall boundary conditions are used at other boundaries. The initial condition for the flow is $(U, 0,0)$. A rectangular control volume is used for lift calculation by using the SLF. The top and bottom faces of the control volume are at $Z= \pm 12 c$. The left face is at $X=-2 c$, and the right face is at $X=X_{\mathrm{TE}}$, where $X_{\mathrm{TE}}$ is the maximum streamwise position of the trailing edge of the airfoil.

\section{B. Flowfields and Unsteady Lift}

The four cases shown in Table 1 are considered for a flapping flat-plate airfoil at $R e=U c / \nu=3 \overline{0} 0$. The low-frequency flapping motion is first studied in cases $\mathrm{A}$ and $\mathrm{C}$, in which the reduced frequency is $k=\pi f c / U=0.06 \pi$. However, in case $\mathrm{C}$, the nondimensional heaving amplitude is $A / c=0.25$ and the time-averaged AOA is $\alpha_{0}=10 \mathrm{deg}$, which are 10 times as large as those in case A. Figures $3 \mathrm{a}$ and $4 \mathrm{a}$ show the vorticity fields around the airfoil at the instants when the lift reaches the maximum and minimum for case A. Although the boundary layer is weakly separated at the sharp leading edge in case $\mathrm{A}$, the shear layers are still close to the upper and lower surfaces of the airfoil in case A, in which the flapping frequency,

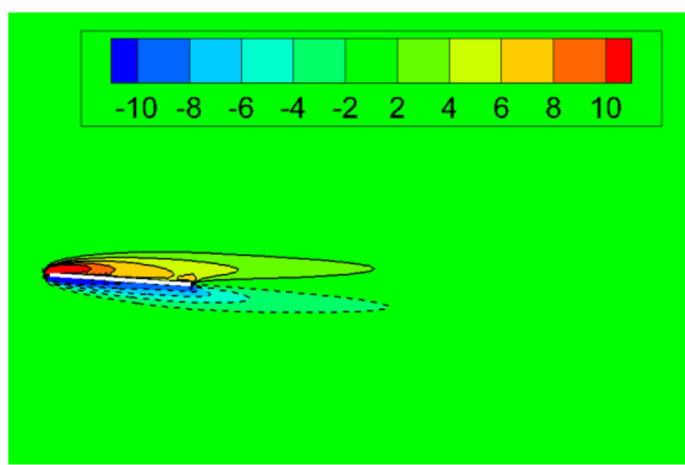

a)

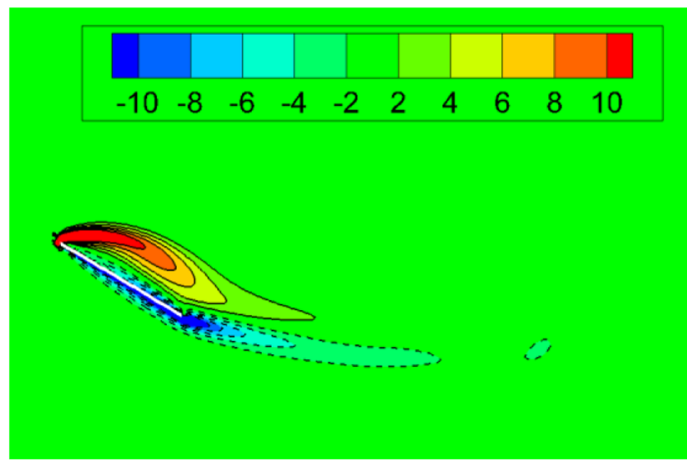

c)

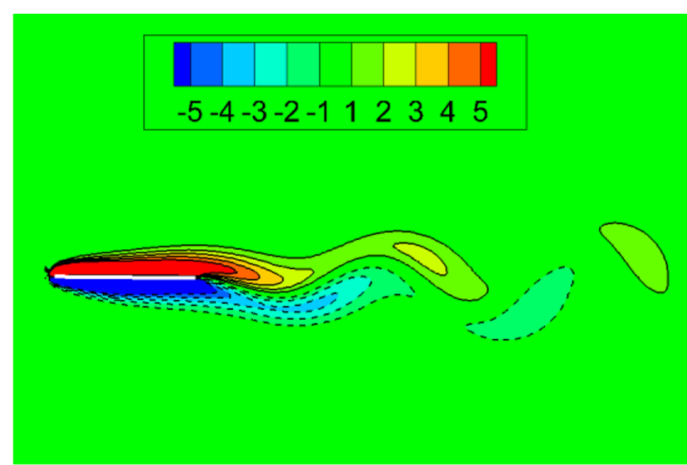

b)

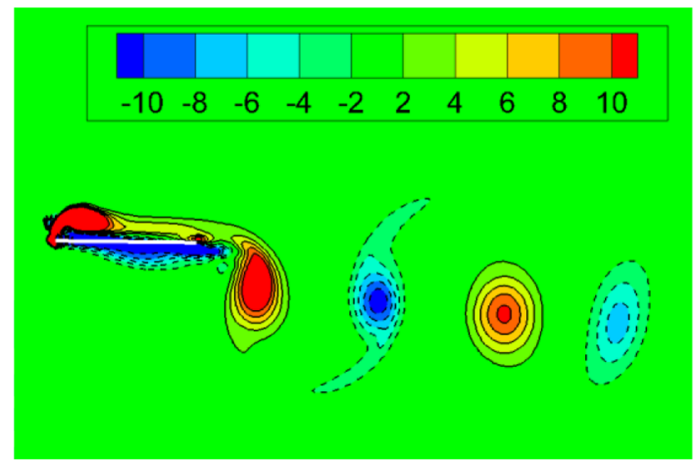

d)

Fig. 3 Vorticity fields around the plate when the lift reaches the maximum in a) case A, b) case B, c) case C, and d) case D. 


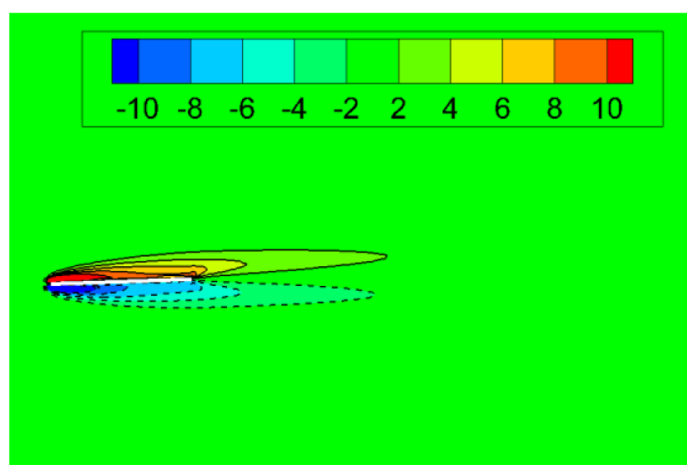

a)

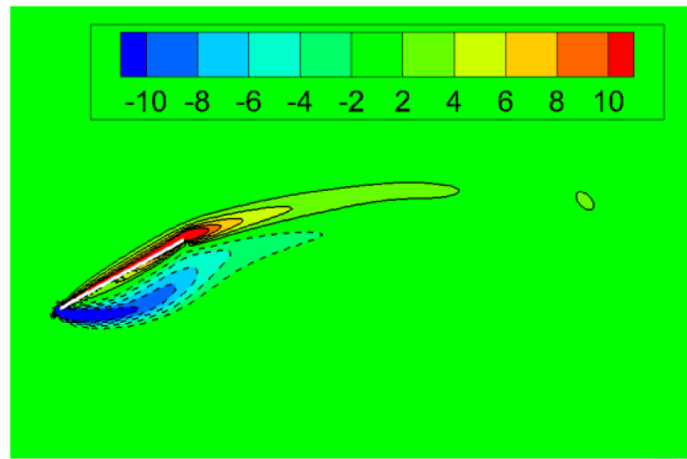

c)

Fig. 4 Vorticity fields around the plate when the lift reaches the minimum in a) case A, b) case B, c) case C, and d) case D.

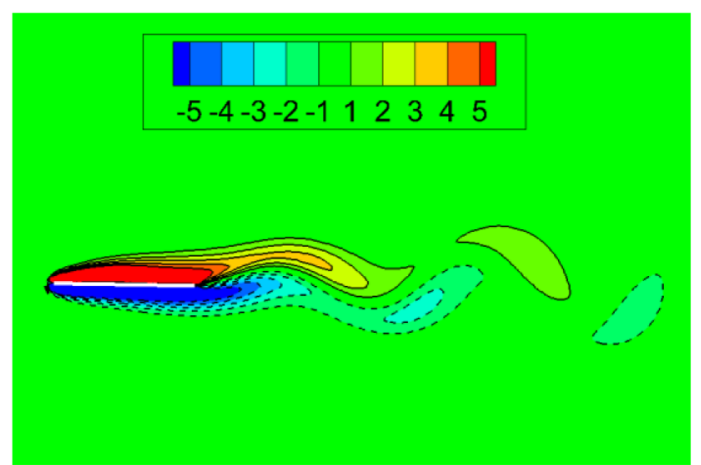

b)

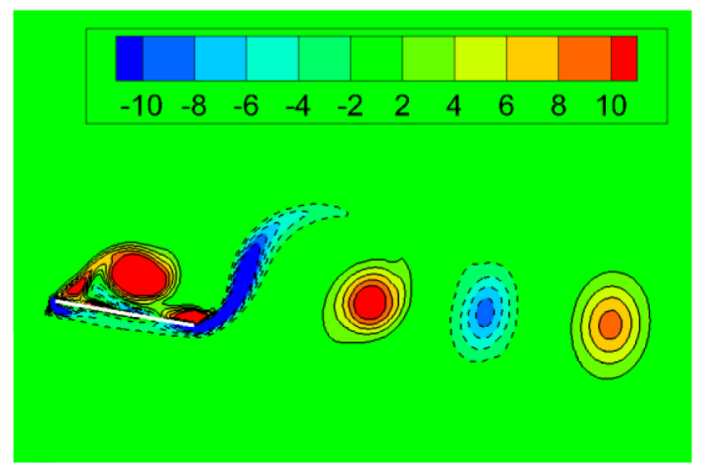

d) heaving amplitude, and time-averaged AOA are small. In contrast, the flow around the airfoil in case $C$ is considerably separated because the heaving amplitude and the time-averaged AOA are much larger. The distributions of the vortex sheet strength $\gamma(x, t)$ along the plate are calculated by using Eq. (8), where the sufficiently large values of $\delta^{+}$and $\delta^{-}$are selected such that the limits of the integral in Eq. (8) are beyond the viscous separated flow domain. Figures $5 \mathrm{a}$ and $5 \mathrm{c}$ show the typical distributions of $\gamma(x, t)$ when the lift reaches the maximum and minimum for cases $\mathrm{A}$ and $\mathrm{C}$. It is noted that the Kutta-Joukowski condition $\gamma\left(x_{\mathrm{TE}}, t\right)=0$ is satisfied only in case $\mathrm{A}$, but it is not met in the other cases where the flow is considerably separated.

The lift coefficient is calculated by using the thin-airfoil lift formula (TALF) [Eq. (10)], based on the distribution of $\gamma(x, t)$. Figure 6 a shows the histories of the lift coefficient given by the TALF and the simple lift formula (SLF) [Eq. (3)] for case A, where the DNS data are obtained by directly integrating the surface pressure and skin friction fields. The SLF is in excellent agreement with the DNS in all the cases. It is expected that the TALF gives the result that is consistent with the DNS data in case A, and the waveform of the lift coefficient remains sinusoidal. More interestingly, as shown in Fig. 6c, the TALF gives a good prediction of the lift coefficient in case $\mathrm{C}$ where the flow around the airfoil is considerably separated. The waveform of the lift coefficient is no longer sinusoidal, indicating that the nonlinear effects associated with the separated flow are significant due to the high-amplitude flapping motion. This indicates that the TALF is still applicable for the separated flows around the airfoil at small Strouhal numbers. As argued by Sears [16], it seems reasonable to use a vortex sheet as a model of a compressed viscous separated flow plus the airfoil. Therefore, the nonlinear effects are intrinsically incorporated into the TALF such that it could be applicable beyond the linear theories.

Furthermore, according to Eqs. (3) and (10), the lift can be decomposed into the vortex lift $\left(L_{\mathrm{vor}}^{\prime}\right)$ and the lift associated with the fluid acceleration $\left(L_{a}^{\prime}\right)$. However, a subtle difference is that $L_{a}^{\prime}$ in the TALF is interpreted as the added-mass lift, which is a reduced case of the SLF. Figure 7 shows the lift coefficients associated with the vortex lift and the fluid acceleration in the TALF and the SLF, where
$C l_{a}=L_{a}^{\prime} /\left(0.5 q_{\infty} c\right)$ and $C l_{\mathrm{vor}}=L_{\mathrm{vor}}^{\prime} /\left(0.5 q_{\infty} c\right)$ are the lift coefficients and $q_{\infty}$ is the dynamic pressure. As shown in Figs. 7a and $7 \mathrm{c}$, the lift coefficients associated with the vortex lift and the fluid acceleration in the TALF and the SLF are consistent in both cases A and $\mathrm{C}$. The time-averaged lift is mainly contributed by the vortex lift $L_{\mathrm{vor}}^{\prime}$, whilereas $L_{a}^{\prime}$ contributes considerably to the temporal variation of the lift coefficient. The vortex lift $L_{\text {vor }}^{\prime}$ given by the TALF is consistent with the SLF in all the cases. This supports the argument made in Sec. III that the nonlinear effects associated with viscous separated flows could be incorporated particularly in the vortex lift in the TALF. However, the lift decomposition reveals that the addedmass lift $L_{a}^{\prime}$ is the main contributor to the error of the TALF, which is particularly large in cases B and D. As indicated in Sec. III, $L_{a}^{\prime}$ in Eq. (10) does not include the effect associated with the unsteady vortical structures in the viscous flow region and the effect of the unsteady boundary $\partial B_{\mathrm{bl}}$. These effects could be significant in massively separated flows. In contrast to the TALF, $L_{a}^{\prime}$ in the SLF includes all the unsteady effects of the flows.

The high-frequency flapping motion is considered in cases B and $\mathrm{D}$, where the reduced frequency is $k=0.6 \pi$. The vorticity fields shown in Figs. 3 and $\underline{4}$ indicate the highly separated flows with energetic vortex shedding from the leading and trailing edges of the airfoil. In case B, where the heaving amplitude is small, as shown in Fig. $7 \mathrm{~b}$, the waveform of the lift coefficient given by the TALF remains basically sinusoidal, which is consistent with the SLF and DNS. However, the result given by the TALF has a phase shift of about 36 deg. As shown in Fig. 7d, in case D where the heaving amplitude is large, the result given by the TALF significantly deviates from those given by the SLF and DNS in both the waveform and phase. This deviation of the TALF from the truth is mainly caused by the added-mass lift $L_{a}^{\prime}$.

\section{Limitations of Linear Models}

Following the derivation of the TALF in Sec. III, we know that the TALF could cope with the nonlinear effects associated with viscous separated flows when the vortex sheet strength distribution $\gamma(x, t)$ is calculated from the vorticity fields obtained from global velocity 


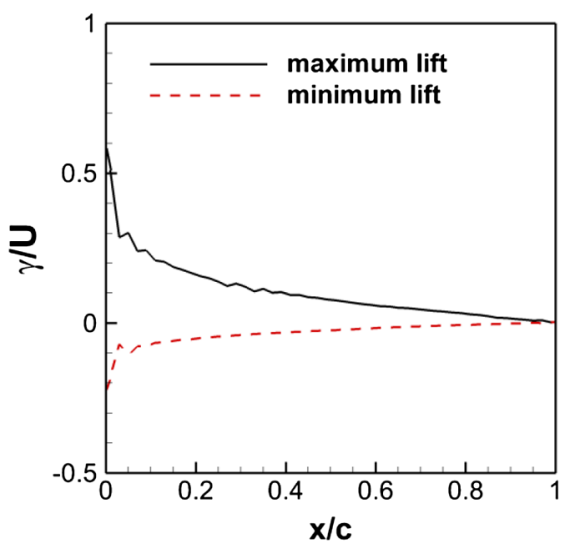

a)

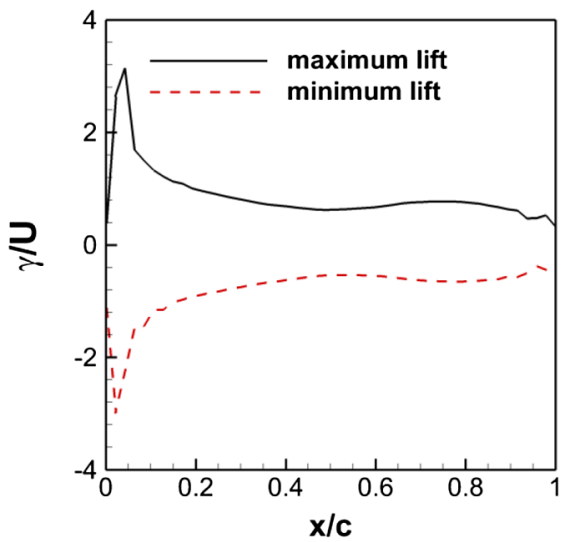

c)

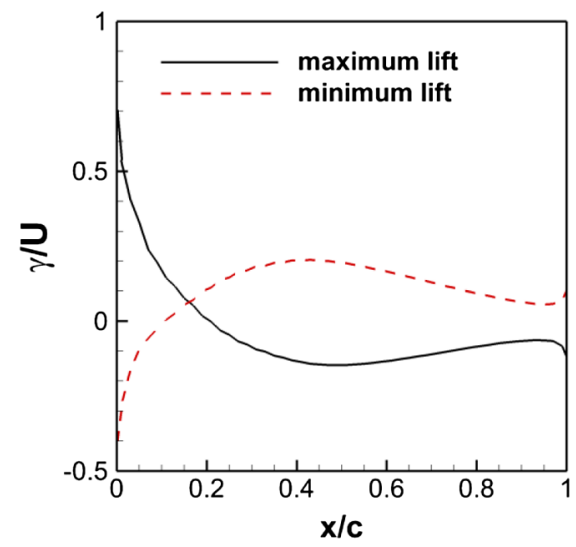

b)

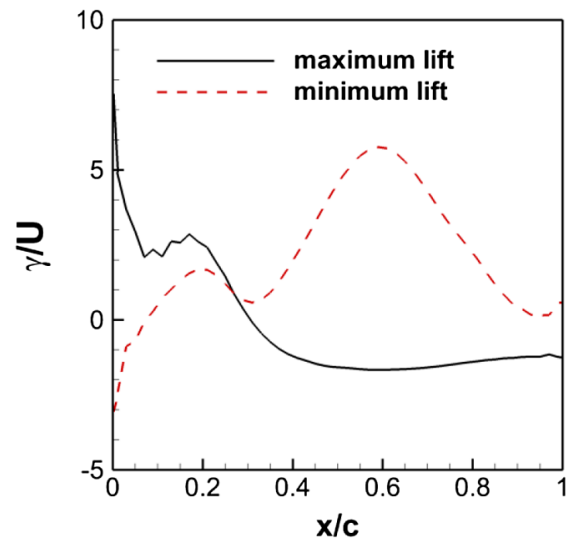

d)

Fig. 5 Distributions of the vortex sheet strength along the plate when the lift reaches the maximum and minimum in a) case $A$, $b$ ) case $B$, c) case $C$, and d) case $D$.
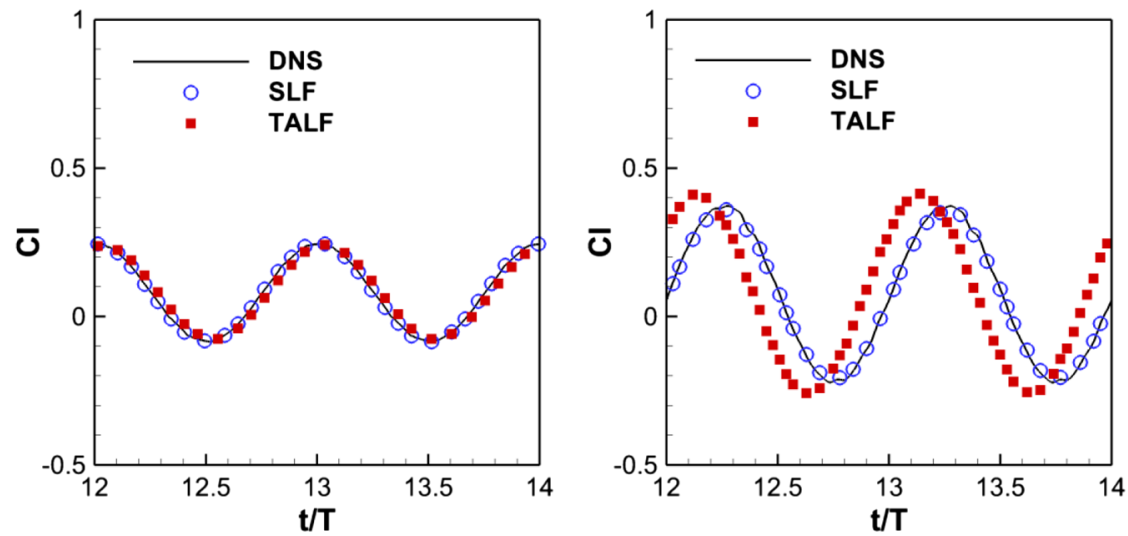

a)

b)
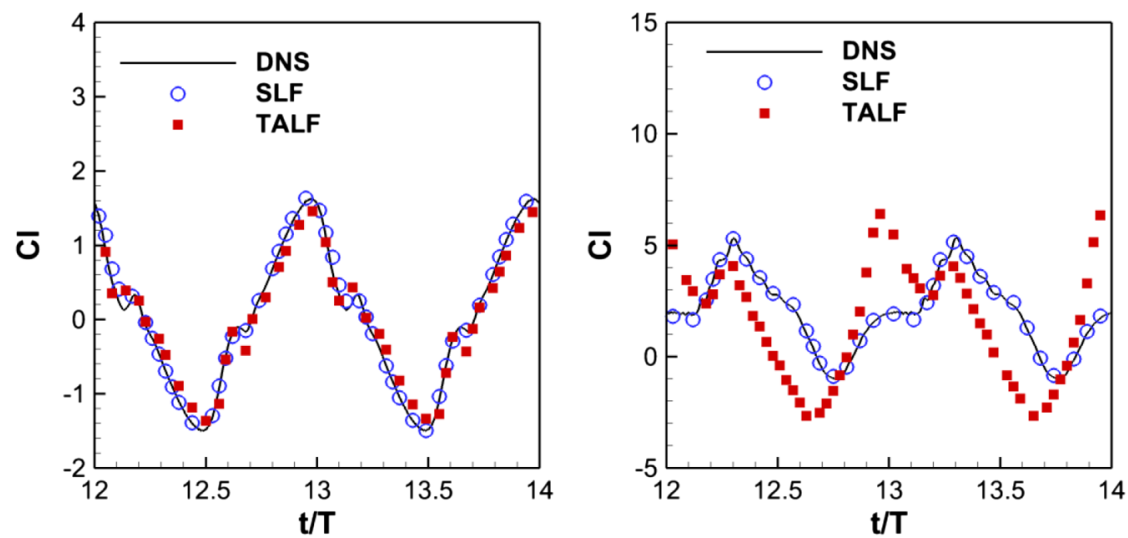

d)

Fig. 6 Lift coefficients given by the TALF, the SLF, and DNS in a) case A, b) case B, c) case C, and d) case D. 

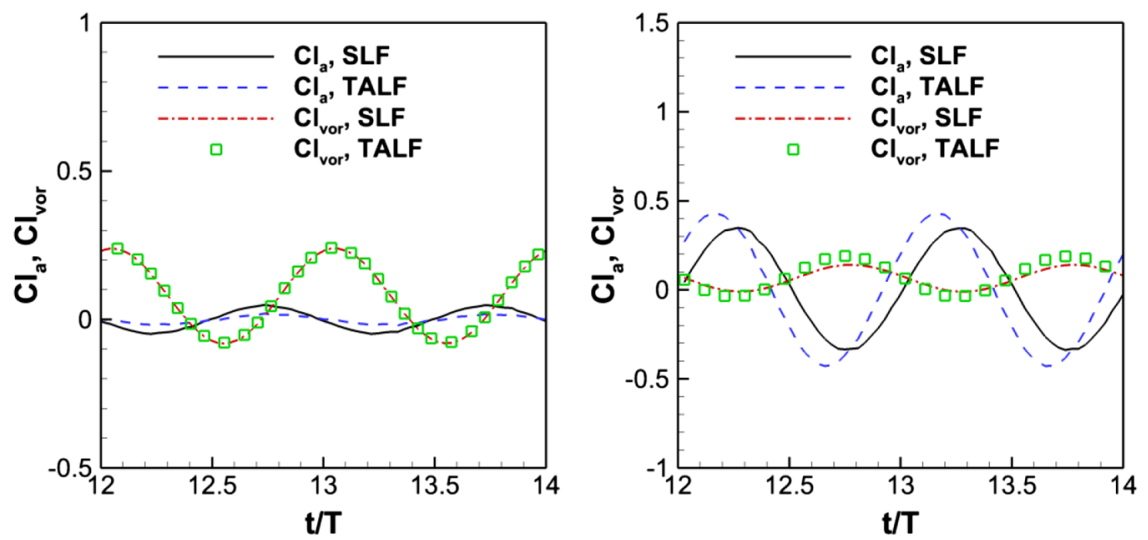

a)

b)
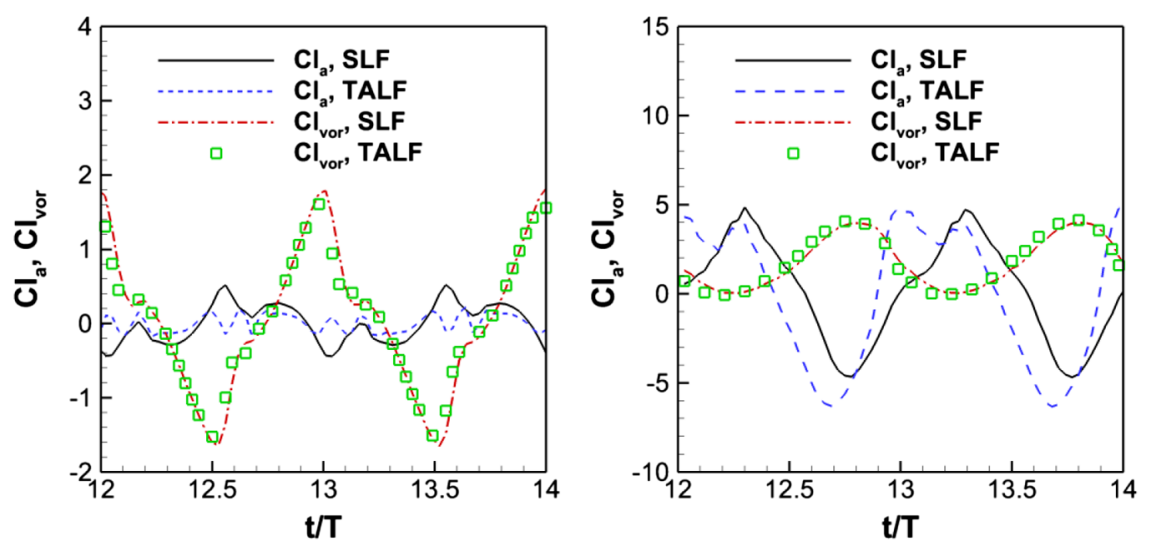

c)

d)

Fig. 7 Lift coefficients associated with the vortex lift and the fluid acceleration in the TALF and the SLF in a) case A, b) case B, c) case C, and d) case D.
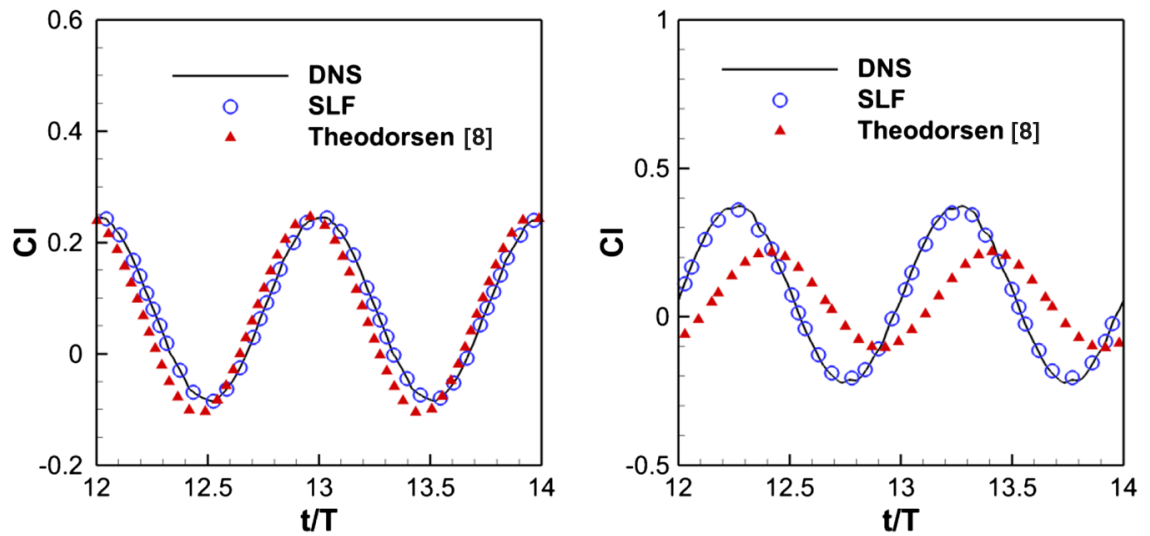

a)

b)
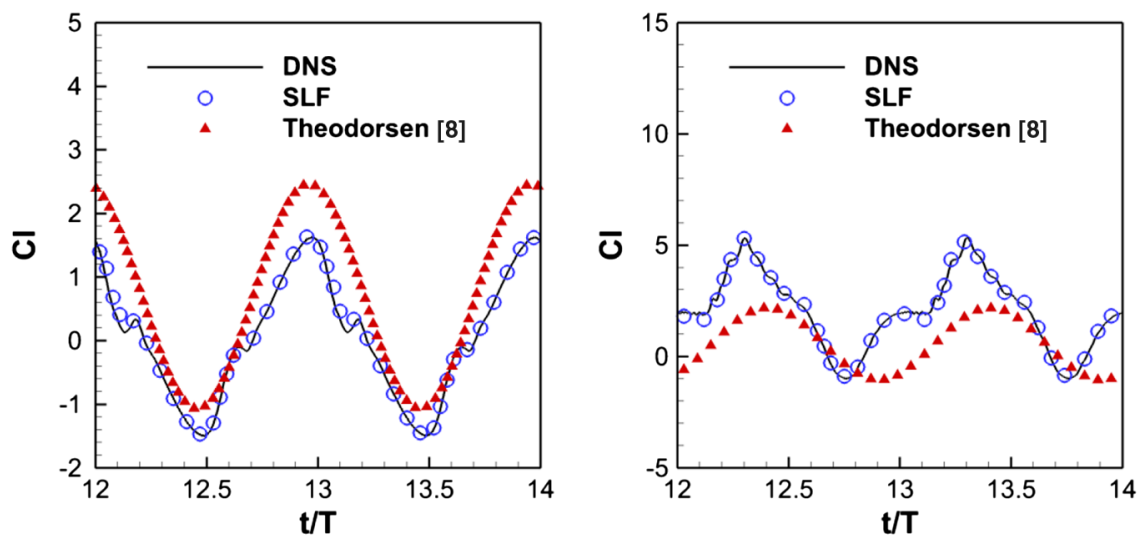

d)

Fig. 8 Lift coefficients given by the Theodorsen lift formula, the SLF, and DNS for a) case A, b) case B, c) case C, and d) case D. 
measurements and CFD. Next, in Sec. IV, the thin-airfoil equation is solved to determine $\gamma(x, t)$ that is decomposed into the quasi-steady part and the wake-induced part. In this step, the linear model for $\gamma(x, t)$ is imposed in the TALF, and thus the von Kármán-Sears lift formula is derived essentially based on the linear model of aerodynamics. Indeed, the examples of steady-state oscillations considered by von Kármán and Sears [6] are the linear cases in which the sinusoidal waveform of the lift remains unchanged. However, the tripe decomposition form of the von Kármán-Sears lift formula is consistent with the TALF and SLF given in the framework of viscous flows. Therefore, as long as $\gamma(x, t)$ is obtained from reliable nonlinear theories, CFD, or measurements, the von Kármán-Sears lift formula is more applicable, in which the nonlinear effects of flows could be naturally incorporated. This is different from other linear unsteady aerodynamic models.

Theodorsen gave a linear aerodynamic model for a harmonically flapping flat plate based on potential flow theory [5]. The Theodorsen lift formula has the noncirculatory and circulatory terms. The noncirculatory term is essentially the added-mass force generated purely by the unsteady motion of the plate. The circulatory term is the quasisteady lift modulated by the Theodorsen function depending on the reduced frequency to take the effect of the infinite straight wake into account. The Theodorsen lift formula used in our case is $\mathrm{Cl}=$ $2 \pi\left(\dot{\alpha}+\ddot{z}_{c}\right)+4 \pi C\left(\alpha+\dot{z}_{c}+\dot{\alpha} / 2\right)$, where $C$ is the Theodorsen function of the reduced frequency $(C=0.514$ and $C=0.633$ for $k=0.6 \pi$ and $k=0.06 \pi$, respectively). Figure $\underline{8}$ shows the lift coefficients given by the Theodorsen lift formula in comparison with those given by the SLF and DNS. Only when the flapping frequency, heaving amplitude, and time-averaged AOA are sufficiently small in case A, the Theodorsen lift formula gives a good prediction. In the other cases, it fails to predict correctly the magnitude, waveform, and phase of the lift coefficient. It is not surprising because the Theodorsen lift formula is strictly linear, which is not applicable for unsteady separated flows.

\section{Conclusions}

Unsteady thin-airfoil theory is revisited by applying the simple lift formula (SLF) that is accurate for a sufficiently large rectangular control volume enclosing a wing in viscous flows. The SLF, which contains the vortex lift (the Lamb vector integral) and the lift associated with the fluid acceleration, allows a direct derivation of the thin-airfoil lift formula (TALF) for a 2-D flowfield decomposed into the outer potential flow and the boundary layer. Accordingly, the TALF contains the Kutta-Joukowski lift and the added-mass lift. This derivation illustrates that the viscous flow around an airfoil is essentially compressed into a vortex sheet with the strength calculated by integrating the vorticity field around the airfoil along the vertical direction. From this perspective, the TALF could be applicable even when the flow around the airfoil is moderately separated. Further, after the vortex sheet strength distribution is decomposed into the quasi-steady part and the wake-induced part, the TALF recovers the classical von Kármán-Sears lift formula. In addition, the analysis based on the SLF gives a direct link between the boundary layer and lift generation. Direct numerical simulations (DNSs) of the flows around a flapping flat-plate airfoil at Reynolds number of 300 are conducted in the four cases with the different flapping frequencies and heaving amplitudes. In the cases with the low reduced frequency $(k=0.06 \pi)$, the lift coefficients given by the TALF are in good agreement with the DNS even when the flow is considerably separated around the flapping airfoil with the large heaving amplitude. In contrast, in the cases with the high reduced frequency $(k=0.6 \pi)$, the predicted lift coefficients by the TALF deviate significantly from the truth in their waveform and magnitude. This deviation of the TALF is mainly caused by the added-mass lift that does not include the effect associated with the unsteady vortical structures in the viscous flow region and the effect of the unsteady boundary-layer edge. These effects could be significant in massively separated flows. Nevertheless, the vortex lift in the TALF is consistent with that in the SLF in all the cases, indicating that the
TALF could cope somewhat with the nonlinear effects associated with viscous separated flows.

\section{Acknowledgments}

This work was supported by the National Natural Science Foundation of China under project noumbers 10872201, 11232011, 11302238, and 11372331, and the National Basic Research Program of China (973 Program) under project number 2013CB834100 (nonlinear science). Tianshu Liu would like to acknowledge the hospitality received at Laboratory of Nonlinear Mechanics during his visit where he accomplished this work. The simulations were performed on TianHe-1. We would like to acknowledge the support from the National Supercomputer Center in Tianjin.

\section{References}

[1] Munk, M. M., "Elements of the Wing Section Theory and of the Wing Theory," NASA Rept. 1050, 1979, pp. 127-149.

[2] Lighthill, M. J., "A New Approach to Thin Airfoil Theory," Aeronautical Quarterly, Vol. 3, Nov. 1951, pp. 193-210.

[3] Wagner, H., "Über die Entstehung des Dyamischen Auftriebes von Tragflügein," Zeitschrift für Angewandte Mathematik und Mechanik, Vol. 5, 1925, pp. 17-35. doi:10.1002/(ISSN)1521-4001

[4] Küssner, H. G., "Zusammenfassender Berricht über des Instationären Auftriebe von Flügein," Luftfahrtforschung, Vol. 13, No. 12, 1936, pp. $410-424$.

[5] Theodorsen, T., "General Theory of Aerodynamic Instability and the Mechanism of Flutter," NACA Rept. 496, 1935.

[6] von Kármán, T., and Sears, W. R., "Airfoil Theory for Non-Uniform Motion," Journal of the Aeronautical Sciences, Vol. 5, No. 10, 1938, pp. $379-390$. doi: $10.2514 / 8.674$

[7] McCroskey, W. J., "Inviscid Flowfield of an Unsteady Airfoil," AIAA Journal, Vol. 11, No. 8, 1973, pp. 1130-1137. doi: $10.2514 / 3.50558$

[8] Edwards, J. W., Ashley, H., and Breakwell, J. V., "Unsteady Aerodynamic Modeling for Arbitrary Motions," AIAA Journal, Vol. 17, No. 4, 1979, pp. 365-374. doi: $10.2514 / 3.7348$

[9] Williams, M. H., "Unsteady Thin Airfoil Theory for Transonic Flows with Embedded Shocks," AIAA Journal, Vol. 18, No. 6, 1980, pp. 615624. doi: $10.2514 / 3.50797$

[10] Leishman, J. G., and Nguyen, K. Q., "State-Space Representation of Unsteady Airfoil Behavior," AIAA Journal, Vol. 28, No. 5, 1990, pp. 836-844. doi: $10.2514 / 3.25127$

[11] Pullin, D. I., and Wang, Z. J., "Unsteady Forces on an Accelerating Plate and Application to Hovering Insect Flight," Journal of Fluid Mechanics, Vol. 509, No. 6, 2004, pp. 1-21. doi:10.1017/S0022112004008821

[12] Gaunaa, M., "Unsteady 2D Potential-Flow Forces on a Thin Variable Geometry Airfoil Undergoing Arbitrary Motion,” Ris $\emptyset$ DTU National Lab. for Sustainable Energy, Rept. R-1478, Roskilde, Denmark, July 2006.

[13] Peters, D. A., "Two-Dimensional Incompressible Unsteady Airfoil Theory-An Overview," Journal of Fluids and Structures, Vol. 24, No. 3, 2008, pp. 295-312. doi:10.1016/j.jfluidstructs.2007.09.001

[14] McCune, J. E., and Tavares, T. S., "Perspective: Unsteady Wing Theory —The Kármán/Sears Legacy," Journal of Fluids Engineering, Vol. 115, No. 4, 1993, pp. 548-559. doi:10.1115/1.2910179

[15] Glauert, H., The Elements of Aerofoil and Airscrew Theory, 2nd ed., Cambridge Univ. Press, Cambridge, England, U.K., 1983, Chap. 7.

[16] Sears, W. R., "Unsteady Motion of Airfoil with Boundary-Layer Separation," AIAA Journal, Vol. 14, No. 2, 1976, pp. 216-220. doi: $10.2514 / 3.7072$

[17] Saffman, P. G., Vortex Dynamics, Cambridge Univ. Press, Cambridge, England, U.K., 1992, Chaps. 3-5.

[18] Wu, J. C., "Theory for Aerodynamic Force and Moment in Viscous Flows," AIAA Journal, Vol. 19, No. 4, 1981, pp. 432-441. doi: $10.2514 / 3.50966$

[19] Quartapelle, L., and Napolitano, M., "Force and Moment in Incompressible Flows," AIAA Journal, Vol. 21, No. 6, 1983, pp. 911- 
913. doi: $10.2514 / 3.8171$

[20] Howe, M. S., "On Unsteady Surface Forces, and Sound Produced by the Normal Chopping of a Rectilinear Vortex," Journal of Fluid Mechanics, Vol. 206, No. 9, 1989, pp. 131-153. doi: $10.1017 / \mathrm{S} 0022112089002259$

[21] Chang, C.-C., "Potential Flow and Forces for Incompressible Viscous Flow," Proceedings of the Royal Society of London. Series A: Mathematical and Physical Sciences, Vol. 437, No. 1901, 1992, pp. 517-525. doi:10.1098/rspa.1992.0077

[22] Wu, J. Z., and Wu, J. M., "Vorticity Dynamics on Boundaries," Advances in Applied Mechanics, Vol. 32, 1996, pp. 119-275. doi:10.1016/S0065-2156(08)70077-2

[23] Noca, F., Shiels, D., and Jeon, D., "A Comparison of Methods for Evaluating Time-Dependent Fluid Dynamic Forces on Bodies, Using Only Velocity Fields and Their Derivatives," Journal of Fluids and Structures, Vol. 13, No. 5, 1999, pp. 551-578. doi:10.1006/jfls.1999.0219

[24] Wu, J.-Z., Pan, Z.-L., and Lu, X.-Y., "Unsteady Fluid-Dynamic Force Solely in Terms of Control-Surface Integral," Physics of Fluids, Vol. 17, No. 9, 2005, Paper 098102. doi:10.1063/1.2055528

[25] Wu, J. Z., Ma, H. Y., and Zhou, M. D., Vorticity and Vortex Dynamics, Springer, Berlin, 2006, Chap. 11.
[26] Wang, S. Z., Zhang, X., He, G. W., and Liu, T., "A Lift Formula Applied to Low-Reynolds-Number Unsteady Flows," Physics of Fluids, Vol. 25 , No. 9, 2013, Paper 093605. doi: $10.1063 / 1.4821520$

[27] Katz, J., and Plotkin, A., Low-Speed Aerodynamics, McGraw-Hill, New York, 1991, Chap. 13.

[28] Estrada, R., and Kanwal, R. P., Singular Integral Equations, Birkhäuser, Boston, 2000, Chap. 3.

[29] Sears, W. R., "Operational Methods in the Theory of Airfoils in NonUniform Motion," Journal of the Franklin Institute, Vol. 230, No. 1, 1940, pp. 95-111. doi:10.1016/S0016-0032(40)90651-2

[30] Wang, S. Z., and Zhang, X., "An Immersed Boundary Method Based on Discrete Stream Function Formulation for Two- and Three-Dimensional Incompressible Flows," Journal of Computational Physics, Vol. 230, No. 9, 2011, pp. 3479-3499. doi:10.1016/j.jcp.2011.01.045

[31] Chang, W., Giraldo, F., and Perot, B., "Analysis of an Exact Fractional Step Method," Journal of Computational Physics, Vol. 180, No. 1, 2002, pp. 183-199. doi: $10.1006 / j \mathrm{cph} .2002 .7087$

M. Choudhari Associate Editor 